\title{
Multiple Linear Regression Models for Partial Discharge Location Along Transformer Windings
}

\author{
Arismar Morais Gonçalves Júnior* Hélder de Paula ${ }^{* *}$ \\ Wallace do Couto Boaventura ${ }^{* * *}$ \\ * Graduate Program in Electrical Engineering, Federal University of \\ Minas Gerais, MG (e-mail: arismarmgj11@gmail.com). \\ ** Faculty of Electrical Engineering, Federal University of Uberlândia, \\ $M G$ (e-mail: drhelderdepaula@gmail.com) \\ *** Electrical Engineering Department, Federal University of Minas \\ Gerais, $M G$ (e-mail: wventura@cpdee.ufmg.br)
}

\begin{abstract}
More recently, it has been noted an increase in the incidence of failures in power transformers, mainly due to degradation of the solid insulation system. The identification of current pulses related to Partial Discharges $(P D s)$ has enabled the early detection and location of the solid insulation deteriorated regions, which is very useful to optimize the planning of the maintenance interventions, preventing unexpected power interruptions and reducing downtime. Even though several techniques for $P D s$ location have been proposed in recent years, limitations can still be noted, such as the inability to specify the discharge location in narrower regions and unproved applicability in windings of different configurations. In view of these, in this work, a $P D$ location method is analysed, based on multiple linear regression models developed in conjunction with stepwise variable selection and analysis of variance methods. The technique is applied from $P D s$ simulation data, obtained through accurate transformer high frequency modelling, using statistical and principal components features of the terminal windings current signals. The results show that the $P D s$ can be effectively located, with high accuracy and success rate, for both layer-type and disk-type windings of transformers.
\end{abstract}

Keywords: Transformers windings; partial discharges; current signals; features extraction; $P D$ location; multiple linear regression.

\section{INTRODUCTION}

Transformers, both distribution and power, are wellconstructed and robust electrical equipment that have high operational reliability and a long useful life. In recent years, however, owing to the steady growth in demand for electricity and volumes of energy transferred, such equipment has been more frequently subjected to harsh operational conditions, such as overvoltages and overloads, which have accelerated its process of deterioration and increased the incidence of failures (Metwally, 2011).

Among the different types of faults that affect transformers, studies show that internal faults in the windings are the most common, around 30\% (Marques et al., 2014). In general, this type of fault is governed by the degradation of the solid insulation system, usually consisting of paper impregnated with oil and/or insulating varnish (Mondal and Kumbhar, 2017).

According to Shuai et al. (2014), in the process of insulation degradation, small imperfections appear on the surface of the insulation, which represent electrically weak regions where the activity of small discharges occur, the socalled Partial Discharges $(P D s)$. Such phenomena usually occur prior to the collapse of the insulation, i.e., it is the precursor of the fault itself, so that, if detected externally, it can be used as an alarm and prevent the evolution of the fault, as well as several inconveniences resulting from the transformer shutdown.

Among the various ways of capturing the activity of the $P D s$, the measurement of current pulses generated by them is highlighted. In this way, high frequency current transformers can be used, installed in the accessible terminals of the windings, i.e., in the phases and neutral/earth terminals (Gonçalves Júnior et al., 2018).

In addition to the identification of the $P D s$, however, an estimation of the locations of these discharges along the windings is also relevant, mainly for repair purposes.

Several techniques for locating $P D s$, based mainly on the high frequency current signals captured at the accessible endings of the windings, have been proposed in recent years. As examples, Nafar et al. (2011) made use of correlation coefficients between waveforms to determine the point of occurrence of the PDs; Homaei et al. (2014) used unsupervised neuro-fuzzy networks, by means of orthogonal transformation of the received signals; Guillen et al. (2016) employed Wavelet-Laplace functions in conjunction with Hellinger's distance calculation; Rahman et al. (2016) used a combination of linear and digital filters, based 
on wavelet coefficients and the main components of the signals; Gonçalves Júnior et al. (2018) applied regression models using statistical features from terminal currents of an experimental winding; among others. Although all these methods present significant performances, with a high percentage of hits for the $P D s$ location, the vast majority was evaluated for only one type of transformer windings, being the applicability in other windings structures thus unknown. In addition, advanced signal processing and classification techniques are normally necessary.

Due to the aforementioned limitations, a methodology for $P D$ localization is proposed in this work, based on multiple linear regression in conjunction with the stepwise variable selection procedure, by means of features extracted from the winding terminal current pulses. The proposed technique utilizes simulated signals, obtained through a high-frequency transformer winding model, to correctly determine, with high accuracy and percentage of hits, the location of the PDs occurrence, both in disk-type and layer-type transformer windings.

\section{MULTIPLE LINEAR REGRESSION}

Multiple Linear Regression $(M L R)$ is a statistical technique that enables the investigation and modelling of the relationship between the variables of a system, where the main objective is to obtain an approximate functional relationship between a certain response of interest $(y)$, or determined output of the system, and a set of independent variables $(x)$, formed by the system input variables.

Although the real format of the mathematical function that relates the independent and dependent variables of a system is usually unknown, polynomial models of (i) first order, (ii) first order with interactions between terms and (iii) second order, defined in equations (1) - (3), respectively, can be used in the majority of applications (Montgomery et al., 2006):

$$
\begin{gathered}
y=\beta_{0}+\sum_{i=1}^{k} \beta_{i} x_{i}+\epsilon, \\
y=\beta_{0}+\sum_{i=1}^{k} \beta_{i} x_{i}+\sum_{i=1}^{k-1} \sum_{j>1}^{k} \beta_{i j} x_{i} x_{j}+\epsilon, \\
y=\beta_{0}+\sum_{i=1}^{k} \beta_{i} x_{i}+\sum_{i=1}^{k} \beta_{i i} x_{i}^{2}+\sum_{i=1}^{k-1} \sum_{j>1}^{k} \beta_{i j} x_{i} x_{j}+\epsilon,
\end{gathered}
$$

where: $\beta s$ are the coefficients of the models, which must be estimated for the models definition; $\epsilon$ represents the measurement and/or modelling errors; and $k$ is the total number of independent variables.

In general, the coefficients of the polynomial models can be adequately estimated by the method of least squares, from a set of $n>k$ observations/measurements of input and output variables of the system in question.

Considering that a set of observations is available, equations (1) - (3) can be rewritten in matrix terms according to (4) and the vector of coefficients of the $M L R$ models $(\boldsymbol{\beta})$ estimated by the least squares method, as in (5):

$$
\begin{gathered}
y=x \beta+\epsilon, \\
\beta=\left(x^{\prime} \boldsymbol{x}\right)^{-1} \boldsymbol{x}^{\prime} \boldsymbol{y} .
\end{gathered}
$$

\subsection{Stepwise Procedure}

An appropriate subset of regressive variables and terms for $M L R$ models can be determined automatically by the stepwise regression technique, which acts to construct these models iteratively. In this regard, statistical tests $F$ and $t$ are normally performed, being some statistical indexes and associated $p$-values calculated and analysed according to the distributions of Fisher-Snedecor and Student, respectively, for a previously established significance level $(\alpha)$. The $p$-values represent the probabilities that the values of the calculated statistical indexes are higher than the related values in the respective distributions.

Stepwise regression is based on forward and backward variable selection methods, which sequentially add or remove the regressive variables to/from the models, respectively. However, since the insertion or exclusion of a variable in $M L R$ models influences the significance of the variables already present or maintained, the models adjusted by the forward and backward methods can be full of redundant variables, which degrades their applicability. In the stepwise method, on the other hand, as each variable is added or removed to/from the model, the significance of variables already present or retained are re-evaluated, aiming to eliminate possible variables that become insignificant.

Figure 1 illustrates the stepwise adjustment procedure of a $M L R$ model, with the establishment of the thresholds $p$-values for the entry or withdrawal of candidate variables and terms, $p_{\text {enter }}$ and $p_{\text {remove }}$, respectively.

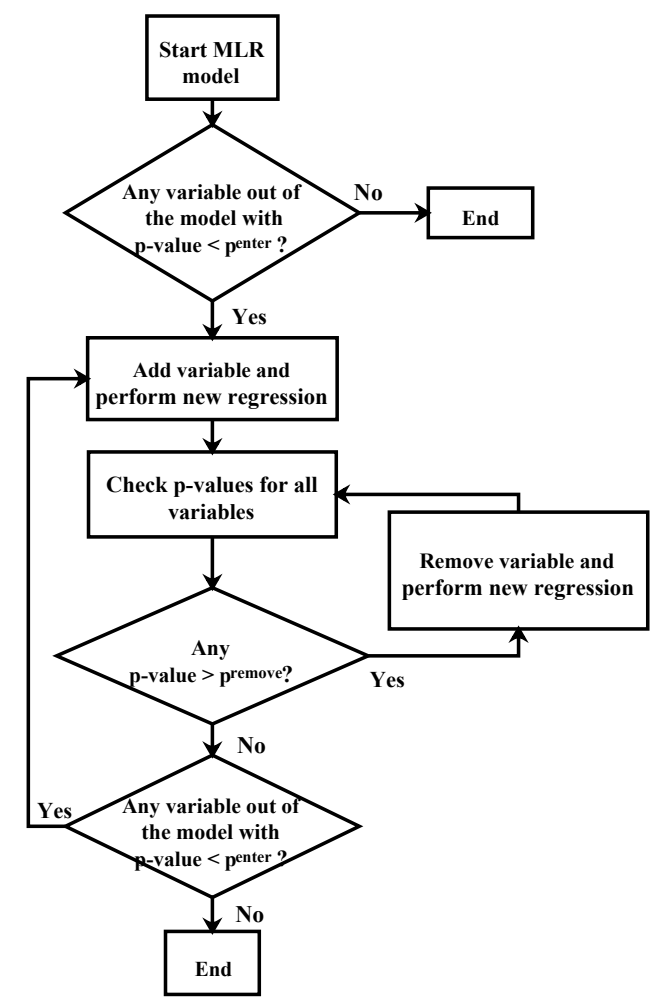

Figure 1. Stepwise regression flowchart.

\section{TRANSFORMER MODELLING AND SIMULATION}

In view of the high frequencies associated with the current pulses of the PDs, the distributed nature of the trans- 
formers windings must be considered in the modelling. In this context, the Multiple Coupled Transmission Lines approach $(M T L)$ is adequate and has been widely used, as in Jafari and Akbari (2007), Hosseini and B. (2017), among others.

In the MTL modelling, each turn of the winding is considered as a transmission line, so that the voltages and currents at any point of the lines/turns can be described by the classical wave equations. System solution is performed by means of its decoupling, with a change of variables by modal quantities and by the application of similarity transformations (Paul, 2008).

For the injection of the $P D s$, before incorporating the boundary conditions of the turns in the resolution of the uncoupled wave equations, the boundary condition of the turn $q-1$, where $q$ is the point of occurrence of the $P D$, must be modified, allowing the injection of the discharge current pulse. With this, transfer functions between possible points of occurrence of the $P D s$ and the winding endings can be determined and, thus, the current pulses at these points.

\subsection{Case Studies}

In this work, the terminal current signals, due to the occurrence of $P D s$ along the windings of the transformers, were generated computationally, using $M T L$ modelling for two different types of windings:

- Case $i$ : winding consisting of several layers of conductors (layer-type);

- Case $i i$ : winding consisting of stacked discs (disktype).

These two configurations represent the main types of windings used in power transformers (Del Vecchio et al., 2010). In distribution transformers, usually the layer-type configuration is utilized.

For the case study $i$, the high voltage winding $(H V)$ of the $15 k \mathrm{VA}, 6600 / 69 \mathrm{~V}$ distribution transformer used in Popov et al. (2007) was selected, consisting of 15 layers of conductors of circular cross section, each composed of 200 turns. The insulation is composed of the conductors insulating varnish and by mineral oil. According to Popov et al. (2007), and as shown in Figure 2(a), the main capacitances required for the $M T L$ modelling are: capacitances between adjacent turns of a same layer $\left(C_{t}\right)$, capacitances between adjacent turns of adjacent layers $\left(C_{L}\right)$, capacitances between the turns of the $H V$ winding first layer and the low voltage winding $\left(C_{W}\right)$ and capacitances between top and bottom turns and the grounded core $\left(C_{g}\right)$.

For case study ii, the $30 M V A, 132 / 20 k V$ power transformer discussed in Jafari and Akbari (2007) is used, whose $H V$ winding is composed of 50 stacked disks, each with 14 turns of rectangular cross section. The insulation system consists of paper impregnated with oil between adjacent turns of the same disk, and structures of paper and oil for the other parts. Figure 2(b) shows the transformer of Jafari and Akbari (2007) with the main capacitances recommended by the authors for the $M T L$ modelling of the $H V$ winding: capacitances between adjacent turns of the same disk $\left(C_{t}\right)$, capacitances between adjacent turns of adjacent disks $\left(C_{D}\right)$ and capacitances between the $H V$ winding bordered turns and the low voltage $(L V)$ and voltage regulating $(V R)$ windings $\left(C_{W}\right)$. Table 1 presents the capacitance values for both transformers:

Table 1 . Windings capacitance values.

\begin{tabular}{ccc}
\hline Capacitance & Case $i$ & Case $i i$ \\
\hline$C_{t}$ & $152.37 p F$ & $1962.17 p F$ \\
$C_{L}$ & $59.29 p F$ & - \\
$C_{D}$ & - & $147.95 p F$ \\
$C_{W(L V)}$ & $2.03 p F$ & $39.02 p F$ \\
$C_{W(V R)}$ & - & $55.71 p F$ \\
$C_{g}$ & $0.50 p F$ & - \\
\hline
\end{tabular}

The inductance and conductance matrices of the transformers $M T L$ models can be derived directly from the capacitance matrices, considering the insulating systems as homogeneous (Paul, 2008). For the calculation of the resistances, the classical equation of conductor resistances, considering the skin effect, can be used. Thus, $R \sim$ $38.37 \sqrt{f} \mu \Omega$ for case $i$ and $R \sim 45.54 \sqrt{f} \mu \Omega$ for case $i$.

\subsection{Simulations}

The MTL modelling of case studies $i$ and $i i$ was implemented in the frequency domain in MATLAB ${ }^{\circledR}$ software. To reduce processing time, each group of 10 turns was lumped for the winding of case $i$ and 2 turns for case ii, according to the procedures described in the works of Popov et al. (2007) and Jafari and Akbari (2007).

The $P D s$ were represented by double exponentials signals, with rise times of $50 \mathrm{~ns}$ and random charge quantities between $-5000 p C$ and $+5000 p C$, relative to partial discharges at the initial stages of solid insulation degradation (Niasar et al., 2015).

For each case study, 50 PDs were injected into each of 10 points distributed over the windings. The simulations were performed for a frequency range from $1 \mathrm{kHz}$ to $20 \mathrm{MHz}$, with a $5 \mathrm{kHz}$ step, being the current signals at the endings of the winding converted to time by the inverse Fourier transform.

Figures 3(a) and 3(b) show the signals obtained for PDs of approximately $+5000 \mathrm{pC}$ occurring in $55 \%$ of the windings of cases $i$ and $i i$, respectively. Here the currents in the "phase" relate to the top endings of the windings, where the transformers are normally supplied, and the currents in the "ground" relate to the grounded terminals, i.e., the last turn of each winding. From the figures, it can be seen that different waveforms result from the $P D s$ in the different winding configurations, with distinct oscillatory characteristics and amplitudes.

\section{MLR MODEL DEVELOPMENT TO PD LOCATION}

The multiple linear regression $(M L R)$ models for the location of the $P D s$ were adjusted from features extracted from the two terminal current signals simulated by $M T L$, which intrinsically hold information related to the discharges occurrence location.

By providing appropriate features for the classification of $P D s$, as discussed in Raymond et al. (2015), statistical features (mean, standard deviation, kurtosis and skewness) 


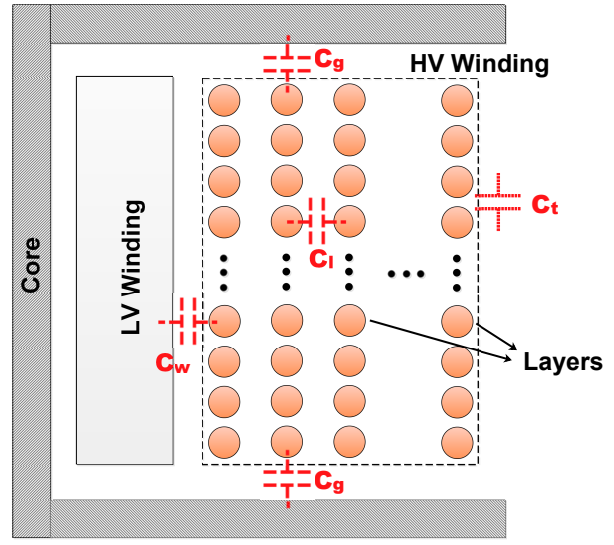

(a)

Figure 2. (a) Layer-type winding; (b) Disk-type winding.

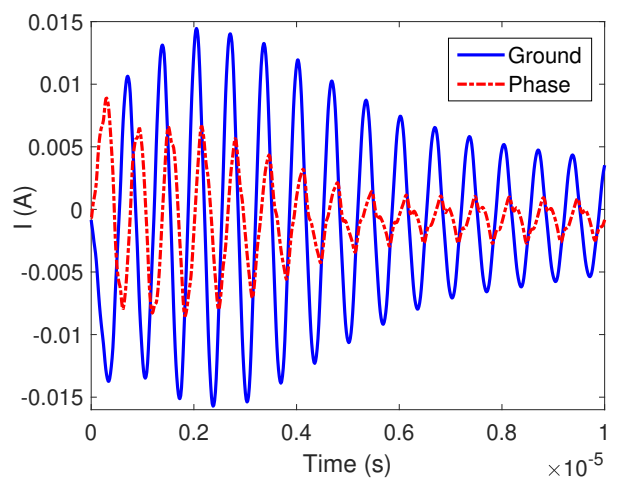

(a)

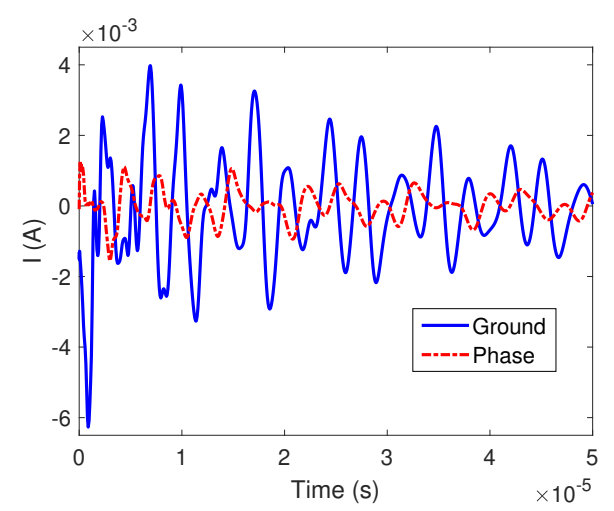

(b)

Figure 3. Terminal currents for case $i$ (a) and case $i i$ (b).

together with energy and apparent charge, and Principal Component Analysis ( $P C A$ ) features, were evaluated in this work, separately. Hence, two different sets of independent variables $x$ were used to obtain the $M L R$ models. The dependent variable $y$, i.e., the output data required for the regression, was defined as the percentage of turns corresponding to each point of injection of the $P D s$. Before the $M L R$ model adjustment, however, the featured variables were separated into two data sets, for the construction (60\%) and validation (40\%), being the latter a fundamental procedure for the response verification of the models for data yet unknown.

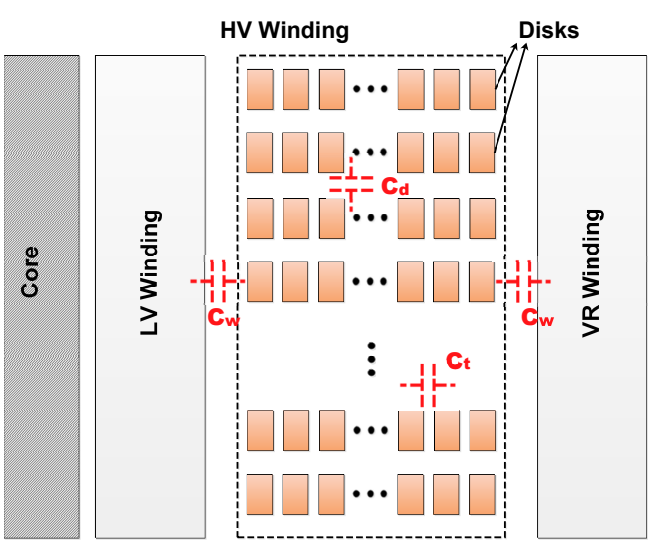

(b)

Among three different polynomials models (equations (1), (2) and (3)) adjusted by the stepwise method and validated, the best model for the location of $P D s$ was determined as the one that presents a better compromise between a higher percentage of hits in validation and fewer terms and variables.

\section{RESULTS}

For each of the case studies considered in this work, $M L R$ models for statistical features, along with energy and apparent charge, and $P C A$ features, were developed separately. As a threshold for the insertion or withdrawal of terms and variables by the stepwise procedure, a level of significance $\alpha=0.05$ was considered. To evaluate the percentage of hits in the validation of the models, an error $\delta= \pm 0.05$ was accepted for the estimated output, which represents a deviation of $\pm 5 \%$ of the actual occurrence points of the PDs.

For comparison purposes, feed-forward Artificial Neural Networks $(A N N s)$ were developed, also in MATLAB ${ }^{\circledR}$ software. For this, the best network topologies were determined as those with the higher hits percentage, varying the number of hidden layers between 1 and 2, the number of neurons in each layer between 5 and 25, and the activation function between linear (purelin), tangent sigmoid (tansig) or logarithm sigmoid (logsig).

Using the statistical features, the best $P D$ localization models were identified as a first-order model with interactions for case $i$, and a second order model for case $i i$. The MLR model of case $i$ consists of 11 linear terms, 41 interactions and 1 constant, whereas the model of case $i i$ contains 8 quadratic terms, 10 linear terms, 23 interactions and 1 constant. Due to space limitations, the extended model equations will not be presented here.

Figures 4(a) and 4(b) show the performance of the $M L R$ models of cases $i$ and $i i$, respectively, when classifying statistical data still unknown by them, i.e., data separated for validation. These data were organized into vectors in descending order from the "phase" terminal, according to the injection points of the $P D s$ in the $M T L$ simulations. Since the vast majority of points (i.e., the locations predicted by the models) are within the expected ranges (considering 
the allowed error threshold of $\pm 5 \%$ ), it confirms that the $M L R$ models for statistical features are able to accurately locate the $P D s$. This is true for the layer-type winding, whose model correctly classified $96.5 \%$ of the validation data, as well as for the disk-type winding, where the model responded with $100 \%$ accuracy.

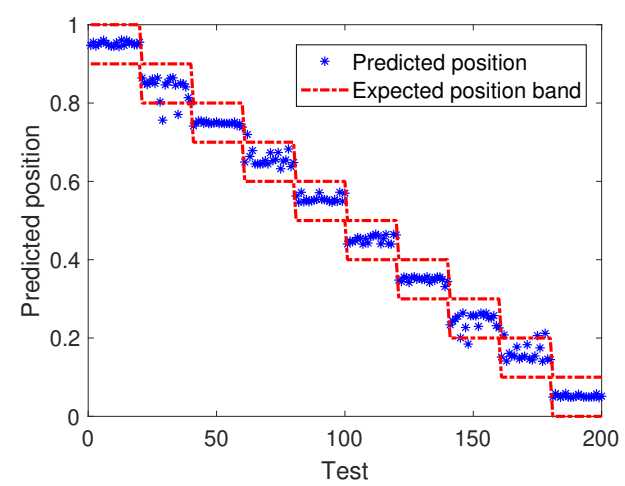

(a)

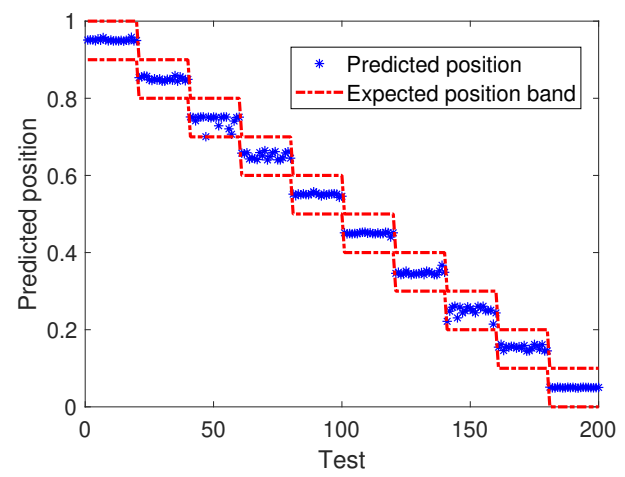

(b)

Figure 4. Validation plot of $M L R$ model for statistical features of case $i$ (a) and case $i i(\mathrm{~b})$.

For each $P D s$ injection point, the hits percentages of the $M L R$ models for statistical features are presented in Table 2 , along with a comparison of these models performance with neural networks. For case $i$, the best $A N N$ was identified as a network with 1 hidden layer of 5 neurons and logsig activation function, capable of correctly locating $92.5 \%$ of the validation data. For case $i i$, the best $A N N$ obtained had 2 hidden layers, each with 10 neurons and tansig activation function, giving $93 \%$ of correct outputs in the validation. In both cases, neural networks presented a worse performance than the regression models, showing that, for statistical features, $M L R$ models are better than the $A N N s$ for the PDs location.

As performed for the statistical features, $M L R$ and $A N N$ models were developed using the $P C A$ features. For cases $i$ and $i i$, the best models obtained were $2 n d$ order, containing only the variables related to the two coefficients of the second component $\left(P C_{21}\right.$ and $\left.P C_{22}\right)$, as represented in equations (6) and (7), respectively:

$$
\begin{gathered}
y=22.85 P C_{22}^{2}+47.24 P C_{22}-25.68 P C_{21}+0.94, \\
y=-1128.26 P C_{22}^{2}-112.67 P C_{22} P C_{21} \\
\quad+2030.95 P C_{22}-813.33 P C_{21}+0.95
\end{gathered}
$$

Table 2. Performance for statistical features.

\begin{tabular}{ccccc}
\hline $\begin{array}{c}\text { PD injection } \\
\text { point }\end{array}$ & \multicolumn{2}{c}{ Case $i$} & \multicolumn{2}{c}{ Case $i i$} \\
\cline { 2 - 5 } & MLR & ANN & MLR & ANN \\
\hline $95 \%$ & $100 \%$ & $100 \%$ & $100 \%$ & $100 \%$ \\
$85 \%$ & $90 \%$ & $100 \%$ & $100 \%$ & $100 \%$ \\
$75 \%$ & $100 \%$ & $100 \%$ & $100 \%$ & $100 \%$ \\
$65 \%$ & $95 \%$ & $100 \%$ & $100 \%$ & $100 \%$ \\
$55 \%$ & $100 \%$ & $60 \%$ & $100 \%$ & $90 \%$ \\
$45 \%$ & $100 \%$ & $100 \%$ & $100 \%$ & $100 \%$ \\
$35 \%$ & $100 \%$ & $65 \%$ & $100 \%$ & $100 \%$ \\
$25 \%$ & $95 \%$ & $100 \%$ & $100 \%$ & $80 \%$ \\
$15 \%$ & $85 \%$ & $100 \%$ & $100 \%$ & $60 \%$ \\
$5 \%$ & $100 \%$ & $100 \%$ & $100 \%$ & $100 \%$ \\
\hline Total & $\mathbf{9 6 . 5 \%}$ & $\mathbf{9 2 . 5 \%}$ & $\mathbf{1 0 0 \%}$ & $\mathbf{9 3 \%}$ \\
\hline
\end{tabular}

Although the $M L R$ models for $P C A$ features have presented satisfactory adjustment parameters for both case studies, they have shown a lower validation performance than the statistical models, as observed in Figure 5.

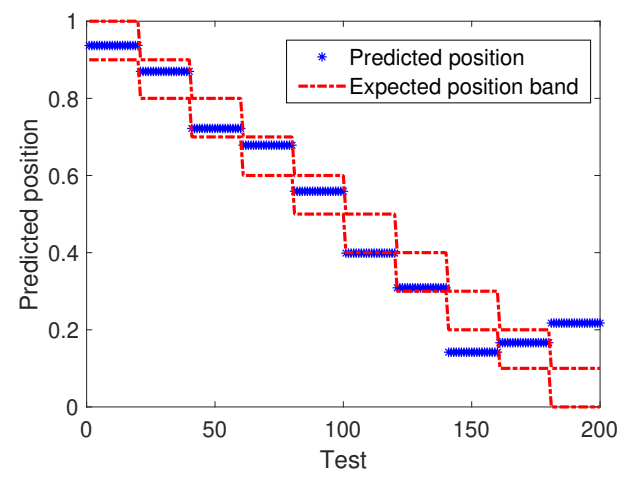

(a)

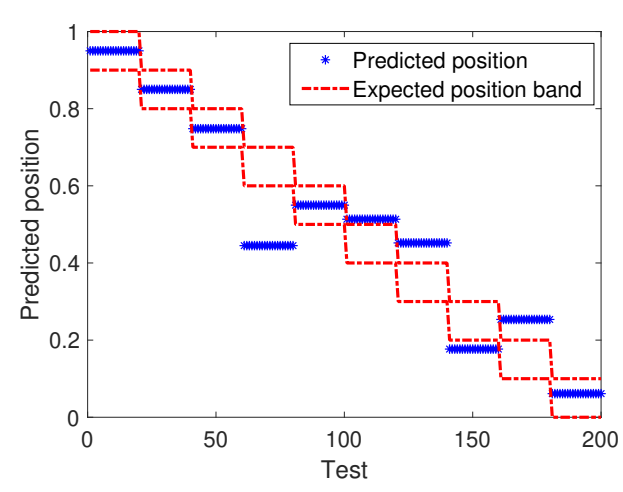

(b)

Figure 5. Validation plot of $M L R$ model for $P C A$ features of case $i$ (a) and case $i i(\mathrm{~b})$.

The best $A N N s$ obtained with the $P C A$ feature variables have similar configurations of those obtained for statistical features. Compared to these networks, the $M L R$ technique presented worse performance, as shown in Table 3.

The inferior performance resulting from the $P C A$, in comparison with the statistical features, can be justified by the fact that $P C A$ provide a worse distinction of the current signals classes, related to the location of the $P D s$. In this sense, $P C A$ appears to be less sensitive to the $P D s$ magnitudes variation. 
Table 3. Performance for $P C A$ features.

\begin{tabular}{ccccc}
\hline $\begin{array}{c}\text { PD injection } \\
\text { point }\end{array}$ & \multicolumn{2}{c}{ Case $i$} & \multicolumn{2}{c}{ Case $i i$} \\
\cline { 2 - 5 } & MLR & ANN & MLR & ANN \\
\hline $95 \%$ & $100 \%$ & $100 \%$ & $100 \%$ & $100 \%$ \\
$85 \%$ & $100 \%$ & $100 \%$ & $100 \%$ & $100 \%$ \\
$75 \%$ & $100 \%$ & $100 \%$ & $100 \%$ & $100 \%$ \\
$65 \%$ & $100 \%$ & $100 \%$ & $0 \%$ & $0 \%$ \\
$55 \%$ & $100 \%$ & $100 \%$ & $100 \%$ & $100 \%$ \\
$45 \%$ & $0 \%$ & $100 \%$ & $0 \%$ & $100 \%$ \\
$35 \%$ & $100 \%$ & $0 \%$ & $0 \%$ & $0 \%$ \\
$25 \%$ & $0 \%$ & $100 \%$ & $0 \%$ & $100 \%$ \\
$15 \%$ & $100 \%$ & $0 \%$ & $0 \%$ & $100 \%$ \\
$5 \%$ & $0 \%$ & $100 \%$ & $100 \%$ & $100 \%$ \\
\hline Total & $\mathbf{7 0 \%}$ & $\mathbf{8 0 \%}$ & $\mathbf{5 0 \%}$ & $\mathbf{8 0 \%}$ \\
\hline
\end{tabular}

\section{CONCLUSIONS}

A methodology for the location of $P D s$ in transformer windings, due to the degradation of the solid insulation system, is analysed in this work. The technique uses statistical or $P C A$ features of the terminal current signals, for the adjustment of Multiple Linear Regression $(M L R)$ models, being the best polynomials determined by the stepwise procedure and hits percentages on validation.

The results show that the proposed $M L R$ technique, when applied with statistical features of the $P D$ current signals, can be effectively employed to locate the discharges for both layer-type and disk-type windings, unlike several other literature methods that have proved applicability only for the latter type. The proposed methodology presented percentages of hits greater than $90 \%$ in the PDs localization, with a $5 \%$ location accuracy. In the same scenario, in comparison with artificial neural networks, the $M L R$ technique presented superior performance.

\section{ACKNOWLEDGEMENTS}

The authors wish to thank the Brazilian agency CAPES, CNPQ and FAPEMIG for the support given to this work.

\section{REFERENCES}

Del Vecchio, R.M., Poulin, B., Feghali, P.T., Shah, D.M., and Ahuja, R. (2010). Transformer Design Principles: With Applications to Core-form Power Transformers. CRC Press - Taylor \& Francis Group, 2nd edition.

Gonçalves Júnior, A., de Paula, H., and do Couto Boaventura, W. (2018). Practical partial discharge pulse generation and location within transformer windings using regression models adjusted with simulated signals. Electric Power Systems Research, 157. doi:10.1016/j.epsr. 2017.12.013.

Guillen, D., Idarraga-Ospina, G., Mombello, E., and Cabral, S. (2016). Partial discharges location in transformer winding using wavelets and Kullback-Leibler divergence. Electric Power Systems Research, vol. 136(1), pp. 398 - 405. doi:10.1016/j.epsr.2016.03.020.

Homaei, M., Moosavian, S.M., and Illias, H.A. (2014). Partial Discharge Localization in Power Transformers Using Neuro-Fuzzy Technique. IEEE Transactions on Power Delivery, vol. 29(5), pp. 2066 - 2076. doi:10. 1109/TPWRD.2014.2339274.

Hosseini, S.M.H. and B., B.P.R. (2017). New high frequency multi-conductor transmission line detailed model of transformer winding for PD study. IEEE Transactions on Dielectrics and Electrical Insulation, vol. 24(1), pp. 316 - 323. doi:10.1109/TDEI.2016. 005694.

Jafari, A.M. and Akbari, A. (2007). Partial discharge location in transformer windings using multi-conductor transmission line. Electric Power Systems Research, vol. $78(6)$, pp. 1028 - 1037. doi:doi:10.1016/j.epsr.2007.08. 004.

Marques, A., de Jesus Ribeiro, C., Azevedo, C.H., dos Santos, J., de Carvalho Sousa, F., and da Cunha Brito, L. (2014). Power transformer disruptions - a case study. IEEE Electrical Insulation Magazine, vol. 30(2), pp. 17 - 21. doi:10.1109/MEI.2014.6749569.

Metwally, I.A. (2011). Failures, Monitoring and New Trends of Power Transformers. IEEE Potentials, vol. 30(3), pp. $36-43$. doi:10.1109/MPOT.2011.940233.

Mondal, M. and Kumbhar, G.B. (2017). Detection, Measurement, and Classification of Partial Discharge in a Power Transformer: Methods, Trends, and Future Research. IETE Technical Review, 1-11. doi:10.1080/ 02564602.2017.1335244.

Montgomery, D.C., Peck, E.A., and Vining, G.G. (2006). Introduction to Linear Regression Analysis. Wiley.

Nafar, M., Niknam, T., and Gheisari, A. (2011). Using Correlation Coefficients for Locating Partial Discharge in Power Transformer. Electrical Power and Energy Systems, Vol. 33, pp. 493 - 499. doi:10.1016/j.ijepes. 2010.11.003.

Niasar, M.G., Taylor, N., Janus, P., Wang, X., Edin, R., and Kiiza, R.C. (2015). Partial Discharges in a Cavity Embedded in Oil-impregnated Paper: Effect of Electrical and Thermal Aging. IEEE Transactions on Dielectrics and Electrical Insulation, Vol. 22(2), pp. 1071 - 1079. doi:10.1109/TDEI.2015.7076808.

Paul, C.R. (2008). Analysis of Multiconductor Transmission Lines. Wiley-Interscience, New York.

Popov, M., der Sluis, L., Smeets, R.P.P., and Roldan, J.L. (2007). Analysis of Very Fast Transients in LayerType Transformer Windings. IEEE Transactions on Power Delivery, vol. 22(1), pp. 238 - 247. doi:10.1109/ TPWRD.2006.881605.

Rahman, M.S., Lewin, P.L., and Rapisarda, P. (2016). Autonomous localization of partial discharge sources within large transformer windings. IEEE Transactions on Dielectrics and Electrical Insulation, 23(2), 10881098. doi:10.1109/TDEI.2015.005070.

Raymond, W.J.K., Illias, H.A., Bakar, A.H.A., and Mokhlis, H. (2015). Partial discharge classifications: Review of recent progress. Measurement, vol. 68, pp. 164 - 181. doi:10.1016/j.measurement.2015.02.032.

Shuai, H., Qingmin, L., Chengrong, L., and Jiangyan, Y. (2014). Electrical and Mechanical Properties of the Oil-paper Insulation under Stress of the Hot Spot Temperature. IEEE Transactions on Dielectrics and Electrical Insulation, vol. 21(1), pp. 179 - 185. doi: 10.1109/TDEI.2013.003967. 